\title{
EMLA Cream vs $10 \%$ Lidocaine Cream for Attenuating Venous Cannulation Pain - A Clinical Trial
}

\author{
Oluwayemisi Bamidele Oluwadun, ${ }^{1}$ Oyebola Olubodun Adekola, ${ }^{2}$ Olufemi I.O. Dada, ${ }^{2}$ Simeon O. Olanipekun, ${ }^{1}$ \\ Adebayo Sulaimon Adetunji, ${ }^{3}$ John Olutola Olatosi, ${ }^{2}$ Olushola Temitayo Kushimo ${ }^{2}$ \\ ${ }^{1}$ Department of Anaesthesia, EKO Hospital, Lagos, Nigeria \\ ${ }^{2}$ Department of Anaesthesia, University of Lagos \& Lagos University Teaching Hospital, Nigeria \\ ${ }^{3}$ Department of Anaesthesia, Lagos State University Teaching Hospital, Nigeria
}

Correspondence to: Dr OB Ogundun, Department of Anaesthesia, Eko Hospital, Ikeja, Lagos, Nigeria; email: gyogundun@yahoo.co.uk

\begin{abstract}
Background: Venous cannulation is a painful procedure that is associated with anxiety, distress and discomfort. But pain is frequently overlooked in adults. Aims and Objective: We compared the efficacy of 5\% EMLA cream and $10 \%$ lidocaine cream in attenuating pain associated with peripheral venous cannulation. Methods: This prospective, randomized, placebo-controlled trial was conducted in 102 ASA I and II adults scheduled for elective surgery. They were randomly allocated by blind balloting to one of three groups: group E had $1.5 \mathrm{~mL}$ of EMLA cream, group L $1.5 \mathrm{~mL}$ of $10 \%$ lidocaine cream, and group P 1.5 $\mathrm{mL}$ KY gel. All cream was applied over a visible vein for 60 min with occlusive dressing. Pain was evaluated using visual analogue scale (VAS) and verbal rating scale (VRS). Results: The mean VAS score was significantly lower with either EMLA cream $(2.62 \pm 1.76 \mathrm{~cm})$ or $10 \%$ lidocaine cream
\end{abstract}

\section{Introduction}

Crile Washington invented the cannula and used intravenous therapy for the management of shock; this management popularized venous cannulation (1). Peripheral venous cannulation is a routine procedure during the delivery of anesthesia, which many adults find painful. Inadequate pain relief is not only unpleasant for patients but may cause anxiety about further treatment, deter patients from seeking medical care in the future (2), and result in increase in heart rate and blood pressure (3). The increase in heart rate and blood pressure leads to increased myocardial oxygen consumption and increased risk of myocardial infarction in patients at risk, especially those with hypertension and coronary artery disease (4). The use of topical anesthetic agents for venous cannulation is on the increase (5). In our institution, analgesia for cannulation is offered only to children. We compared the efficacy of topical 5\% EMLA cream and 10\% lidocaine cream in attenuating pain associated with peripheral venous cannulation in adults.
$(1.85 \pm 1.58 \mathrm{~cm})$ than with placebo $(4.78 \pm 1.88 \mathrm{~cm}), \mathrm{p}<0.001$. Most patients who received EMLA cream (76.5\%) or $10 \%$ lidocaine cream (70.6\%) compared with placebo (55.9\%) had mild pain during cannulation using VRS. Conclusion: The eutectic mixture of local anesthetic cream and lidocaine cream attenuated pain associated with peripheral venous cannulation to varying degrees.

Key words: Venous cannulation pain, 10\% lidocaine, EMLA, VAS, VRS, Side effects

Ann Afr Surg. 2019; 16(1):4-10

DOI: http://dx.doi.org/10.4314/aas.v16i1.2

Conflicts of Interest: None

Funding: None

(C) 2019 Author. This work is licensed under the Creative Commons Attribution 4.0 International License.

\section{Methods}

This prospective, randomized, placebo-controlled study was conducted between April 2013 and March 2014 after institutional approval and informed consent were obtained from all patients. Adult patients aged 18-60 years, with the American Society of Anesthesiology (ASA) physical status score I or II, scheduled for elective procedures under anesthesia were studied. Criteria for exclusion were: refusal to participate, past history of hypertension, arrhythmias, chronic pain, and local skin infection, those on antiarrhytmic agents such as calcium channel blockers and beta blockers, analgesic use 24 hours before venous cannulation, allergy or sensitivity to amide local anesthetic and glycerine, and more than one attempt at cannulation. Power was analysed for independent $t$ test to determine an appropriate sample size; the difference in mean of the visual analogue scale during venepuncture from a previous study was 13.3 (6). To achieve a power of $80 \%$ and $\alpha=0.05$, a minimum sample size of 34 was considered appropriate. 


\section{Study protocol}

Routine preoperative anesthetic was review the day before surgery. The study protocol was explained to the patients and they were educated on the use of the visual analogue scale (VAS) and verbal rating scale (VRS) that were used to assess pain. The primary outcome compared the effects of $10 \%$ lidocaine cream and of EMLA cream on VAS and VRS. The secondary outcome compared the effects of $10 \%$ lidocaine cream and of EMLA cream on the heart rate, blood pressure and cutaneous skin manifestation.

In the operating theatre, the researcher randomly placed the patients into three groups; each patient picked a prewritten card from a bag. Group E treatment consisted of EMLA cream, Group L 10\% lidocaine cream and Group P used KY gel; the placebo cream (KY gel) is reported not to have an analgesic effect (7). The patients and research assistant were blinded to the cream used. A multiparameter monitor was attached to the patient to measure heart rate, non-invasive blood pressure and oxygen saturation before and during cannulation. The proposed cannulation site was cleaned with alcohol swab, and the research assistant applied $1.5 \mathrm{~mL}$ of the appropriate cream over the dorsum of the patient's hand. Next, an improvised occlusive dressing made of 4 by 4 gauze was applied and held in place with plaster for 60 $\mathrm{min}$. The gauze was removed after $60 \mathrm{~min}$ and the researcher assessed skin sensations such as itching, burning, tingling and cold, after which the cream was removed gently using a methylated spirit swab.

For uniformity, the researcher performed venous cannulation using an $18 \mathrm{G}$ cannula on all patients, and the cannula was secured with transparent plaster. Each patient's reaction during cannulation was noted by a second research assistant and reported as expressionless, grimacing, hand withdrawal, shouting or crying. Each patient was observed for features of vaso-vagal reaction such as dizziness, light headedness, sweating, or nausea.

Each patient was then asked to grade the cannulation pain on the VAS, a $10-\mathrm{cm}$ line marked 0 at one end and 10 at the other $(0=$ no pain; $10=$ worst pain imaginable $)$. Assessments of pain on the linear scale were measured to the nearest centimeter. Each patient also assessed pain using the VRS as none, mild, moderate, and severe.

Each patient's immediate post-cannulation heart rate and blood pressure were measured once the cannula was secured with transparent plaster, then at every 1-minute interval for 10 minutes: before cannulation (baseline)-TB, immediate post cannulation (T0), 1 min after cannulation (T1), 2 min after cannulation (T2), 3 min after cannulation (T3), 4 min after cannulation (T4), $5 \mathrm{~min}$ after cannulation (T5), $6 \mathrm{~min}$ after cannulation (T6), 7 min after cannulation (T7), 8 min after cannulation (T8), 9 min after cannulation (T9), and 10 min after cannulation (T10).

The cannulation site was continuously observed for local skin reactions such as blanching, erythema, localized induration, edema and urticaria hourly for the first 2 hours and then at 6 hours, 12 hours, and 24 hours post cannulation. Allergic reactions, if any, were noted and treated with standard rescue measures.

\section{Statistical analysis}

Demographic, blood pressure, heart rate and visual analogue scores were analyzed using analysis of variance, while clinical data and VRS were analyzed with test of proportions and Chi-square test; $\mathrm{p}<0.05$ was considered statistically significant. Data obtained were analyzed using the Statistical Package for Social Sciences (SPSS) Version 21 (SPSS Inc., Chicago, IL, USA).

\section{Results}

A total of 102 adult surgical patients were recruited. The mean age, weight, height and BMI were comparable between the 3 groups (Table 1).

Table 1: Demographic and clinical characteristics of patients ( $n=34$ for each group)

\begin{tabular}{lcccc}
\hline & \multicolumn{3}{c}{ Treatment groups } & \\
\cline { 2 - 4 } Variable & EMLA & Lidocaine & Placebo & p value \\
\hline $\begin{array}{l}\text { Age/years } \\
\text { (mean } \pm S D)\end{array}$ & $32.4 \pm 10.0$ & $35.9 \pm 9.2$ & $35.4 \pm 9.3$ & 0.27 \\
$\begin{array}{l}\text { Weight/kg } \\
\text { (mean } \pm S D)\end{array}$ & $75.1 \pm 9.67$ & $73.5 \pm 14.6$ & $74 \pm 13.6$ & 0.88 \\
Height (m) & $1.7 \pm 0.77$ & $1.65 \pm 0.07$ & $1.6 \pm 0.03$ & 0.79 \\
$\begin{array}{l}\text { BMI (kg/m²) } \\
\text { Gender ratio }\end{array}$ & $27.8 \pm 3.4$ & $27.1 \pm 6.2$ & $27.6 \pm 5.1$ & 0.83 \\
(M:F) & $13: 21$ & $20: 14$ & $8: 26$ & 0.26 \\
$\begin{array}{l}\text { ASA (ratio } \\
\text { I:II) }\end{array}$ & $19: 15$ & $20: 14$ & $24: 10$ & 0.23 \\
\hline
\end{tabular}

\section{Pain scores}

The median VAS score at venous cannulation differed significantly between the three groups: group E $2(0-8)$, group L $2(0-5)$ and group P $5(1-8), \mathrm{p}<0.001$. VAS score between groups $\mathrm{E}$ and $\mathrm{L}$ was similar, $\mathrm{p}=0.45$. On distribution of pain between the group using VRS, more patients with pain were in group $\mathrm{P}$ (34) than in groups $\mathrm{E}$ (32) and L (26), $\mathrm{p}<0.001$; pain distribution was similar between groups $\mathrm{E}$ and $\mathrm{L}, \mathrm{p}=0.45$. 


\section{Reactions during cannulation}

Table 2 shows the frequency distribution of patients with different reactions to cannulation. In group E, $13(38.2 \%)$ patients were expressionless during cannulation while $19(55.9 \%)$ grimaced. In group L, more than half of the patients $18(52.9 \%)$ were expressionless while $14(41.2 \%)$ grimaced. In group $\mathrm{P}, 6(17.6 \%)$ of the patients were expressionless and 16(47.1\%) grimaced. The difference in reactions during cannulation across the 3 groups was statistically significant, $\mathrm{p}=0.003$.

Table 2: Mean visual analogue scale scores in the three groups ( $n=34$ for each group)

\begin{tabular}{lcccl}
\hline & EMLA & Lidocaine & Placebo & p value \\
\hline Mean \pm SD & $2.6 \pm 1.8$ & $1.9 \pm 1.5$ & $4.8 \pm 1.9$ & $0.001^{*}$ \\
Median & 2.0 & 2.0 & 5.0 & \\
\hline
\end{tabular}

* statistically significant

\section{Heart rate}

Figure 1 shows the mean heart rates against time in the different groups. The mean heart rate in group $\mathrm{E}$ rose from baseline value at $\mathrm{T} 0$. The mean heart rate at T3 min fell below baseline and remained steady below baseline value throughout the study period. In group $\mathrm{L}$, mean heart rate fell below baseline value immediate post cannulation (T0) and remained below baseline value till the end of the study. The lowest value in heart rate in group $\mathrm{L}$ was at $\mathrm{T} 4$. The mean heart rate in the placebo group rose above baseline value and remained above baseline value throughout the study period.

Figure 2 shows percentage changes in mean heart rates from baseline values with time. In groups $\mathrm{E}$ and $\mathrm{P}$, a rise of $1.78 \%$ and $7.17 \%$ was observed when baseline values were compared with immediate postcannulation values (T0). Group L demonstrated a fall of $-0.72 \%$ from baseline values at (T0). The placebo group demonstrated the highest change with a $7.17 \%$ rise from baseline values. Significant differences were observed in percentage changes in mean HR from baseline values between the groups during the study except at T8, $\mathrm{p}=0.10$.

\section{Mean arterial pressure}

Figure 3 shows the mean arterial pressure (MAP) against time. MAP decreased in all the groups at T3 with that of the treatment groups dropping below baseline values. Figure 4 shows the mean percentage change in mean arterial pressure from baseline values over the study period. MAP increased in all the 3 groups immediate post cannulation (T0). The increases from baseline values were $2.92 \%, 2.10 \%$ and $6.01 \%$ in groups $\mathrm{E}, \mathrm{L}$ and $\mathrm{P}$ respectively. These changes were not statistically significant, $p=0.39$. Throughout the study period there was no statistical difference between the 3 groups when considering percentage change in MAP from baseline values.

\section{Mean rate pressure product}

Figure 5 shows the mean rate pressure product over the study period. Statistical difference was observed only at T3,

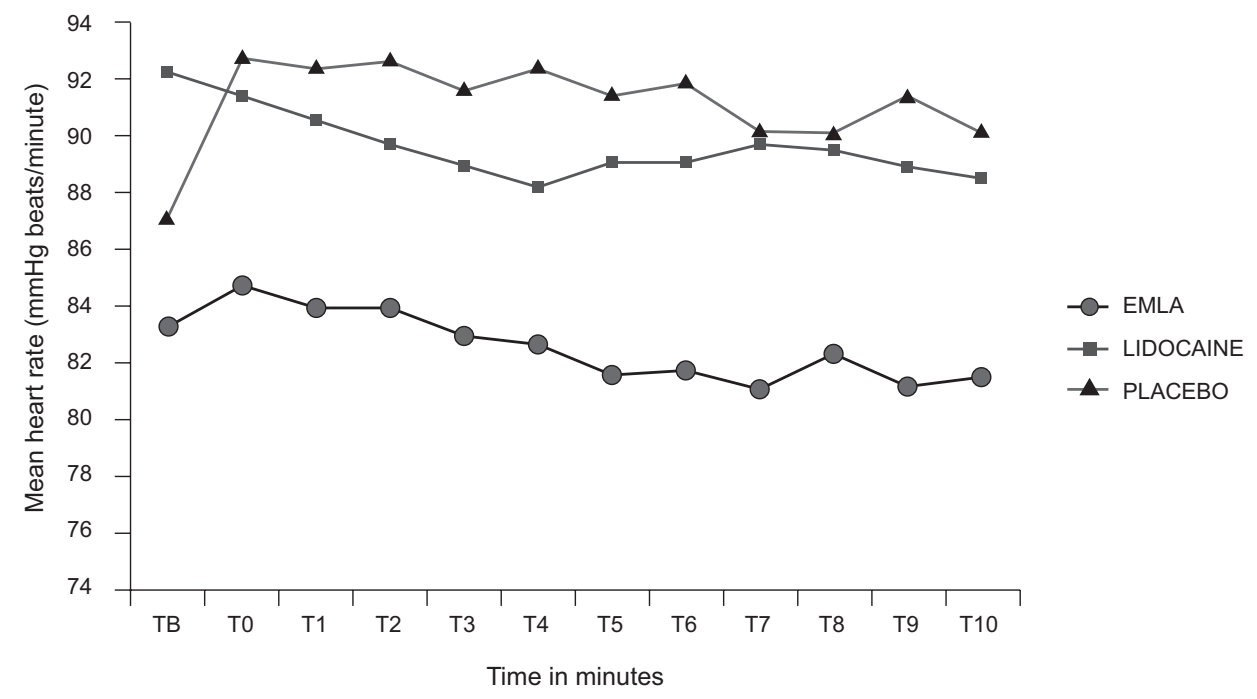

Figure 1: Mean heart rate $(\mathrm{bpm})$ at different time points

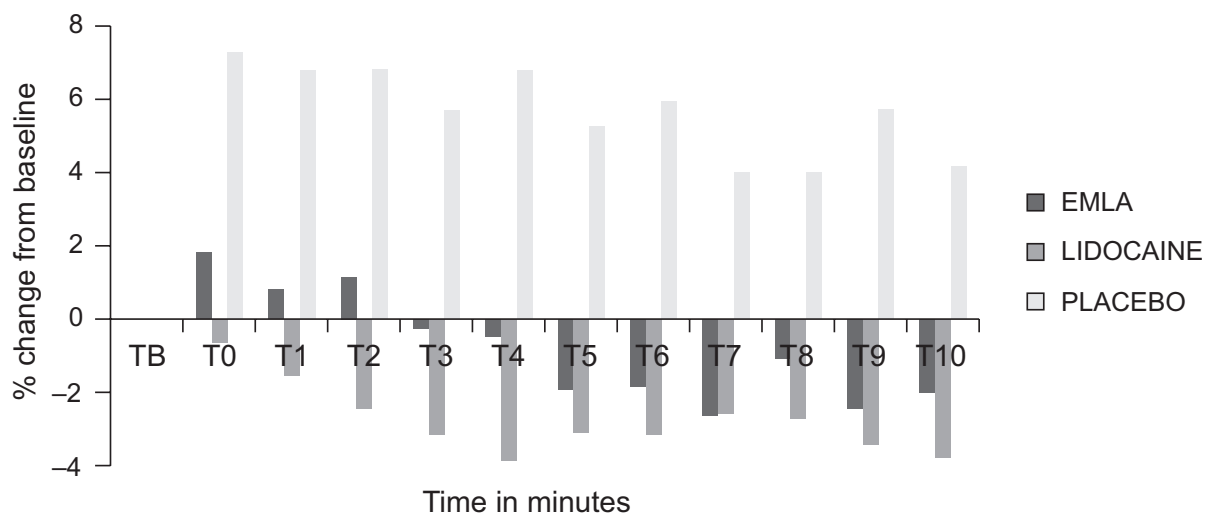

Figure 2: Percentage change in mean heart rate from baseline 
$\mathrm{p}=0.01$. Group $\mathrm{P}$ showed the highest rise of $12,365 \mathrm{mmHg}$. beat. $\mathrm{min}^{-1}$ in the immediate post cannulation period while the least rise was in the EMLA group at 5,997.1 $\mathrm{mmHg}$. beat. $\mathrm{min}^{-1}$ at $\mathrm{T} 7$ post cannulation.

\section{Side effects}

In group E, 1 patient (2.9\%) had burning skin sensation; in group L, 2 patients (5.9\%) had burning skin sensation and 1 patient (2.9\%) tingling sensation. In assessing local skin reactions in all 3 groups, 1 patient (2.9\%) in each group was observed to have blanching of the skin when treatment creams ceased. Blanching was no longer observed 1 hour post cannulation.

\section{Discussion}

We have demonstrated that VAS was lower after application of EMLA and $10 \%$ lidocaine cream $60 \mathrm{~min}$ before venous cannulation than after application of KY gel. However, VAS was comparable with EMLA and 10\% lidocaine cream. A similar observation with EMLA and lidocaine creams was reported earlier; however, a comparison was not made with the placebo (8). The observation in the two studies may suggest that lidocaine and EMLA creams have equipotent analgesic effect during venepuncture, while KY gel had inferior analgesic property. Miller et al. compared the analgesic effect of SC 1\% lidocaine (0.3-0.5 $\mathrm{mL}), 2.5 \mathrm{~g}$ EMLA cream and $1 \mathrm{~mL}$ Iontocaine (a mixture of $2 \%$ lidocaine and 1:100,000 epinephrine) on pain at application of agent and during venous cannulation in adults scheduled for ambulatory surgery (5). The authors observed that at application of topical anesthetic agent, patients who received SC $1 \%$ lidocaine had a higher VAS (15.6 \pm 9.4$) \mathrm{mm}$ than those receiving either EMLA cream (VAS $0.2 \pm 0.4$ ) $\mathrm{mm}$ or

Figure 5: Mean rate pressure product at different time points 
Iontocaine (VAS $2.5 \pm 4$ ) mm. During venous cannulation, patients who received EMLA cream had a higher VAS $(22.3 \pm 20.1) \mathrm{mm}$ than those who had either SC $1 \%$ lidocaine (VAS 6.4 \pm 8.5 ) $\mathrm{mm}$ or Iontocaine (VAS $4.2 \pm 7.2$ ) $\mathrm{mm}$. The authors concluded that Iontocaine (Numby Stuff system using ionotophoresis) had superior analgesic effect during application and at venous cannulation. This is because ionotophoresis uses a mild electrical current to deliver the ions of medication and drive medication through the skin, hence ensuring that the block is localized and dense. The limitation of Miller et al.'s study was the relatively small size of 10 individuals per group; future studies are encouraged with larger sample sizes (5).

In another study among adult volunteers, either EMLA or 4\% lidocaine cream was shown to be effective in reducing pain associated with pinprick when compared with placebo (9). However, the anesthetic efficacy of $4 \%$ lidocaine cream and EMLA cream was comparable, though the use of occlusive lidocaine had a faster onset of action than EMLA cream. Similarly, no adverse events were seen (9). The authors concluded that a topical preparation with $4 \%$ lidocaine is an effective and safe treatment option for superficial anesthesia, with faster onset of action.

Selby and Bowles (10) compared the analgesic effect of three agents. Patients were randomly allocated to 4 groups before venous cannulation: SC lidocaine, ethyl chloride spray, EMLA cream applied for $5 \mathrm{~min}$, and control (no analgesia). Pain during venepuncture was assessed using a VAS of 100 $\mathrm{mm}$. They observed that lidocaine and ethyl chloride were very effective in removing venepuncture pain $(95 \%$ CI for the difference between the control and spray groups was $7.0-23 \mathrm{~mm}$, and between the control and lignocaine groups 10-25 mm). EMLA cream had a limited, but insignificant, effect (95\% CI for difference between control and EMLA groups was $2-15 \mathrm{~mm}$ ). They concluded that the analgesic effect of lignocaine and ethyl chloride was better than that of EMLA cream applied for $5 \mathrm{~min}$ before venous cannulation (10). This is contrary to our observation with lidocaine and EMLA creams; the difference may be because EMLA was applied $60 \mathrm{~min}$ before venepuncture in our study.

In another study (11), the VRS in patients who received EMLA cream 60 min before cannulation was lower than VRS with lidocaine iontophoresis $(20 \mathrm{~mA})$ during venous cannulation with a large bore catheter (18G). However, the onset of analgesia was short with iontophoresis; similarly, erythema and paraesthesia were transient and common (11). The authors concluded that EMLA provided a dense and superior analgesia than ionotophoresis. This was contrary to the observation made by Miller et al. (5). The former researchers attributed their observations to the use of a low iontophoretic dose. The variation in these observations may reflect a different patient group, different iontophoretic dose and a smaller stimulus $(5,11)$.

The difference in mean pain score in studies on pain during venous cannulation has been attributed to differences in size of cannula, site of venous cannulation, dosage and duration of application of topical agent, use of premedication, and gender $(2,9,10,12)$.

\section{Hemodynamic parameters}

We observed a significant increase in heart rate immediate post cannulation (T0) from baseline values in groups $\mathrm{E}$ and $\mathrm{P}$ but a decrease in heart rate in group $\mathrm{L}$.

Similarly, a $1.5 \%$ decrease in heart rate in patients treated with lidocaine injection prior to cannulation was reported earlier (4), despite the difference in the route of administration and concentration of lidocaine in the two studies. While this study used $4 \%$ lidocaine cream, the latter study administered $0.25 \mathrm{~mL}$ of $1 \%$ lidocaine directly in the subcutaneous layer (4).

A prospective, randomized clinical trial assessed the efficacy and side effects of commonly used topical anesthesia methods in adults receiving peripheral venous cannulation. The study was double-blinded to the degree that the methodologies allowed. One hundred and fifty healthy adults undergoing elective surgery were randomly allocated to five groups: EMLA cream, ethylchloride spray, intracutaneous infiltration with $2 \%$ lidocaine, placebo cream, and no treatment. Venepuncture was performed with a $18 \mathrm{G}$ cannula on the dorsal side of the hand. Puncture pain and pain caused by the topical treatment itself were measured using VAS (range: 0-100 mm). Hemodynamic response, difficulties in performing the puncture and side-effects were recorded. All analgesic techniques were well tolerated. Hemodynamic response and degree of puncture difficulty showed no differences between the groups. Puncture pain (median mm VAS) following infiltration (1.0) and EMLA (10.0) was significantly lower than in no treatment (30.0) or in placebo (30.0). The benefit of local infiltration was altered by injection pain (11.5). Spray did not significantly lower puncture pain (26.5) and, in addition, was associated with discomfort (10.5). In adults, EMLA cream significantly reduces puncture pain and represents an acceptable alternate method for topical anesthesia in venous cannulation. Local lidocaine infiltration is impaired by applicational pain, whereas spraying the puncture site with ethylchloride has no analgesic benefit.

The percentage change in mean heart rate at cannulation in 
group $\mathrm{P}(7.17 \%)$ is comparable with that in the placebo group $(7.25 \%)$ in the latter study (3). However, the percentage increase in mean HR was sustained for $10 \mathrm{~min}$ in our study but for $3 \mathrm{~min}$ in another study (4). We attributed our observation to lack of premedication while some researchers premedicated all their patients with $20 \mathrm{mg}$ temazepam one hour before venepuncture (4). On the contrary, a decrease in heart rate $15 \mathrm{~min}$ post cannulation following the use of EMLA cream before cannulation was reported in another study (13). The observed decrease was recorded $15 \mathrm{~min}$ post cannulation, which may not be a good representation of what occurred immediate post cannulation.

We reported a sudden increase in MAP at the 10th min (T10) only in group E patients. The percentage change from baseline value in MAP at this time (T10) was also increased. This increase may be due to reduced analgesic effect with early manifestation in the MAP. It is possible that other hemodynamic parameters may also have shown an increase had the duration of study been extended. In this study, $10 \%$ lidocaine cream provided a consistent protective effect against significant increase in heart rate and MAP accompanying venous cannulation. These effects may not be unrelated to the degree of pain attenuation that lidocaine delivers. $10 \%$ lidocaine gave the lowest pain scores and also the lowest level of alteration in hemodynamic variables.

Langham et al. (4) observed a $10-15 \%$ increase in MAP in the placebo group. In this study, MAP rose by $6.01 \%$ in group P. This disparity may be due to the different sites of blood pressure measurement. Langham et al. measured blood pressure on the finger using the finapres (4), while we measured at the upper arm using an electronic sphygmomanometer. The finapres technique has been shown to have low precision for systolic and MAP measurements, and is thus not permitted for absolute blood pressure measurements in individual patients (14). Finger arteries are affected by contraction and dilatation in response to psychological and physical stress and these in turn affect the readings from the finapres (14). An awake patient must be relaxed for accurate readings to be obtained from the finapres (14). An electronic sphygmomanometer with an appropriate size cuff applied to the upper arm is more likely to give readings close to those obtained were invasive monitoring used (14). Therefore, the finapres might have given an exaggerated MAP in the placebo group.

The rate-pressure product (RPP: a product of systolic blood pressure and heart rate) is a good index of myocardial oxygen consumption, and the maximum recommended range is 12,000 to $23,000 \mathrm{mmHg}$.beat. $\mathrm{mm}^{-1}$ (14). Values above this are commonly associated with myocardial ischemia and angina. In our study, the mean RPP throughout in the placebo group was 10, $970 \mathrm{mmHg}$.beat. $\mathrm{mm}^{-1}$, in the EMLA group $10,328 \mathrm{mmHg}$.beat. $\mathrm{mm}^{-1}$, and in the lidocaine group 11,881 mmHg.beat. $\mathrm{mm}^{-1}$. None of the groups had RPP range that fell within the maximum range expected; as such, there may not be significant increase in oxygen consumption in normotensive patients following venous cannulation.

In the perioperative period, Rao et al. (15) recommend not greater than $20 \%$ fluctuation in heart rate and systolic blood pressure in patients with cardiac morbidity going for noncardiac surgery. The increase in baseline values observed of $7.17 \%$ for heart rate and $6.01 \%$ for MAP in the placebo group in response to venous cannulation were lower than the allowable fluctuation. These values were further reduced in the two treatment groups E (HR, 1.78\%; MAP, 2.92\%) and L (HR, 0.72\%; MAP, 2.10\%). This implies that with the attenuation of venous cannulation pain, hemodynamic fluctuations can be minimized. Therefore, in patients with cardiac morbidity (those with hypertension and coronary artery disease) who may show exaggerated hemodynamic response to pain, it is imperative that these fluctuations are avoided by attenuating cannulation pain.

\section{Side effects}

We observed minimal side effects on application of treatment creams. During cream application 1 patient in the EMLA group and 2 patients in the lidocaine group complained of burning. Tingling sensation was also reported by 1 patient in the lidocaine group. Other authors, however, did not have similar experience with lidocaine application (4). The sensations reported by these patients subsided once the creams were removed. Browne et al. (6) reported mild pruritus following the use of amethocaine.

Blanching of the skin after removal of treatment creams was observed in 1 patient in each group. A higher incidence had been reported earlier in a similar study; in the EMLA cream group, 3 patients $(8.8 \%)$ had blanching of skin while 10 patients $(29.8 \%)$ had erythema (16). EMLA cream is known to cause initial local vasoconstriction, leading to blanching; this is followed by vasodilatation, resulting in erythema and induration (17). We reported no erythema and only 1 patient blanched, this we attributed to the dark skin of our studied population unlike other studies with lightskinned patients. Kano et al. (18) noticed local erythema in 8 out of 24 patients treated with lidocaine for venous cannulation pain. Browne et al. (6) compared EMLA with topical Amethocaine for cannulation and observed that blanching occurred more with the EMLA group (23 patients out of 32 patients) while erythema was observed more in the 
Amethocaine group (11 patients out of 32). The cutaneous manifestation observed resolved within an hour. Sakamoto et al. (16) observed a reduction in the number of patients with local reaction $30 \mathrm{~min}$ after cream removal (blanching from $8.8 \%$ to $2.9 \%$, erythema from $29.4 \%$ to $8.8 \%$ ), suggesting that these cutaneous manifestations are local and transient. This study is limited by the paucity of literature on $10 \%$ lidocaine for comparison and critical analysis with our study. Nevertheless, we have demonstrated that EMLA and 10\% lidocaine creams attenuated pain and the associated pressor response to venous cannulation to varying degrees. Lidocaine cream gave lower pain score and better cardiovascular stability during cannulation than EMLA cream. Both were associated with transient, localized cutaneous manifestations.

\section{References}

1. Herman RE. Shock, physiological surgery and George Washington Crile: Innovation in the progressive era. Ann Surg. 1981; 194:121-2.

2. Bond M, Crathrone L, Peters JL, et al. First, Do No Harm: Pain relief for the peripheral venous cannulation of adults - a systematic review and network meta-analysis. BMC Anesthesiol. 2016; 16(1):81.

3. Jagadamba A, Karthiyanee K, Shankar MS, et al. Gender variation in pain perception after intravenous cannulation in adults. IJA.2011; 28(1).

4. Langham BT, Harrison DA. The pressor responsetovenous cannulation: Attenuation by prior infiltration with local anaesthetic. Br J Anaesth. 1993; 70:519-21.

5. Miller KA, Balakrishman G, Eichbauer G, et al. $1 \%$ lidocaine injection, EMLA cream, or 'Numby Stuff' for topical analgesia associated with peripheral intravenous cannulation. AANA Journal. 2001;69(3):185-7.

6. Browne J, Awad I, Plant R, et al. Topical Amethocaine (AmetopTM) is superior to EMLA for intravenous cannulation. Can J Anaesth. 1999; 46:1014-18.

7. Li HWR, Wong CYG, Lo SST, et al. Effect of local lignocaine for pain relief during suction termination of first trimester pregnancy: A randomized controlled trial. Hum Reprod. 2006;
21:1461-6.

8. Armagan E, Kocabas E, Koksal O, et al. Comparison of the efficacies of topical anaesthetics in the reduction of the pain during peripheral intravenous cannulation: A randomised trial. Hong Kong J. Emerg Med. 2012; 19(3):183-8.

9. Herberger K, Krause K, Maier K, et al. Local anesthetic effects of lidocaine cream: Randomised controlled trial using a standardised prick pain. J DermTreat. 2012; 23(6):437-42.

10. Selby I, Bowles BJ. Analgesic for venous cannulation: A comparison of EMLA (5 minutes application), lignocaine, ethyl, and nothing. J R Soc Med. 1995; 88:264-7.

11. Moppett IK, Szypula K, Yeoman PM. Comparison of EMLA and lidocaine iontophoresis for cannulation analgesia. Eur J Anaesthesiol. 2004; 21(3):210-3.

12. Macario A, Weinger M, Troung $\mathrm{P}$, et al. Which clinical anaesthesia outcomes are both common and important to avoid? The perspective of a Panel of Experts' Anaesthesiologists. Anesth Analg. 1999; 88:1085-91.

13. Speirs AF, Taylor KH, Joanes DN, Girdler NM, et al.A randomised, double-blind, placebo-controlled comparative study of topical skin analgesics and the anxiety and discomfort associated with venous cannulation. Br D J. 2001; 190:444-9.

14. Imholz BPM, Weiling W, Montfrans GAV, et al. Fifteen years' experience with finger arterial pressure monitoring: Assessment of the technology. Cardiovasc Res. 1998; 38:605-16.

15. Rao TLK, Jacobs KH. Reinfarction following anaesthesia in patients with myocardial infarction. Anesthesiology. 1983; 59:499-04.

16. Sakamoto M, Kano T, Sadanaga M, et al. Dermal patch anaesthesia: Comparison of $10 \%$ lignocaine gel with absorption promoter and EMLA cream. Anaesthesia 1993; 48:390-2.

17. Yeoh CN, Lee CY. Pain during venous cannulation: Doubleblind, randomized clinical trial of analgesic effect between topical Amethocaine and eutectic mixture of local anaesthetic. J Anaesthesiol Clin Pharmacol. 2012; 28:205-9.

18. Kano T, Hashiguuchi A, Nakamura M, et al. Comparative study of transdermal $10 \%$ lidocaine gel with and without glycrrhentinic acid monohemiphthalate disodium for pain reduction at cannulation. Anaesth Analg. 1992; 74:535-8. 